\title{
Diseño de las articulaciones naturales
}

\author{
ANGULO CARRERE MªT., LLANOS ALCAZAR L.F.*, PUERTA FONOLLA A.J.*
}

\author{
Departamento de Enfermería H. *Departamento de Ciencias Morfológicas. Universidad Complutense de Madrid
}

Desde un punto de vista teórico, todas las articulaciones móviles del cuerpo humano podrían realizar un total de 6 movimientos sobre los tres ejes del espacio: 3 Translaciones (una en cada eje) y 3 Rotaciones (cada una sobre cada eje) dando lugar a articulaciones con seis grados de libertad de movimiento, pero su diseño anatómico, en especial la forma de sus superficies articulares y la existencia de potentes ligamentos que bloquen el movimiento articular, van a limitar varios de estos seis grados de libertad.

Según su diseño anatómico y el movimiento que realizan sobre los tres planos anatómicos de referencia, las diartrosis han sido divididas, de forma clásica, en seis grandes grupos:

\section{- Enartrosis \\ - Trocleartrosis \\ - Trocoides \\ - Condiloartrosis \\ - Encaje recíproco \\ - Artrodia}

\section{ENARTROSIS}

Corresponde a una articulación de tipo ESFÉRICA, establecida entre una esfera maciza incluida en una superficie hueca. Posee movimiento sobre los tres ejes del espacio y, un ejemplo de este tipo de articulación lo constituyen la unión escapulo-humeral y la articulación coxo-femoral.

En su mayoría, las articulaciones móviles del cuerpo humano no sólo están diseñadas para realizar determinados movimientos, sino que como en el caso de la cadera y el resto de las articulaciones que constituyen la extremidad inferior, su especial diseño facilita el soporte de las solicitaciones mecánicas a las que son sometidas.

Este tipo de articulaciones están sujetas a cargas prolongadas durante el mantenimiento estático de la posición inmóvil y, durante toda la fase de apoyo de la marcha. Dado que esta sobrecarga representa una desventaja fisiológica para el cartílago articular, la geometría de los elementos que forman la unión articular va a ser tal que, la superficie de contacto de cada extremo articular va a variar durante el desarrollo del movimiento. Esto implica una INCONGRUENCIA de las superficies articulares denominada por los autores anglosajones «HigherPair Connections» 1 .
Este mecanismo descrito anteriormente se observa en la articulación coxo-femoral. Teniendo en cuenta que en las superficies curvas la presión media ejercida es proporcional no al área de la superficie sino a la de su proyección en un plano, esta situación, en el caso de la cadera, provocaría una hiperpresión en la zona central de la cúpula acetabular (Fig. 1). Para evitar dicha sobresolicitación, existe una incongruencia entre las superficies articulares de forma que, a medida que aumenta la carga (Fig. 2) la esfera, en este caso la cabeza femoral, se introduce más en la cavidad del acetábulo, produciéndose el contacto articular con toda la cúpula cuando las furezas soportadas por la articulación son iguales superiores a la mitad del peso del individuo ${ }^{2}$.

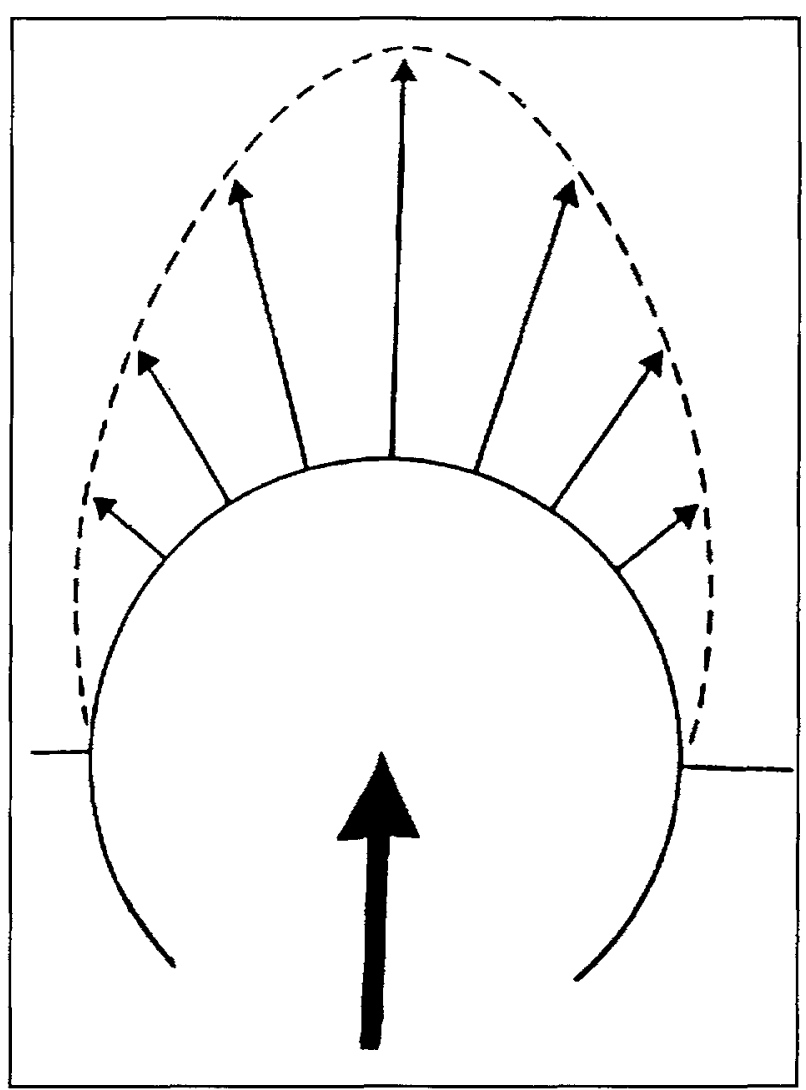

Fig. 1. Representación gráfica del reparto de fuerzas en las superficies curvas (Owen R, Goodfellow J, Bullough P. Fundamentos Científicos de Ortopedia y Traumatología. Barcelona. 1984.) 


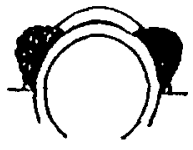

( LEVE)

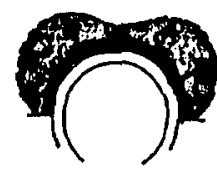

(MODERADA)

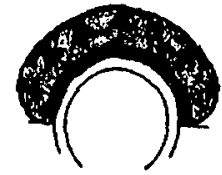

( IGUAL\%PESO)
Fig. 2. Esquema gráfico en el que se observa la congruencia de las superficies articulares en relación a la carga $(O$ wen $R$, Goodfellow J, Bullough P. Fundamentos Científicos de Ortopedia y 'Traumatología. Salvat, Barcelona. 1984).

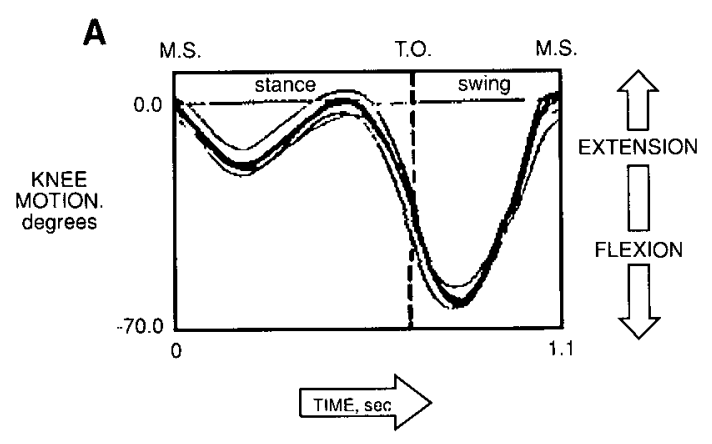

B

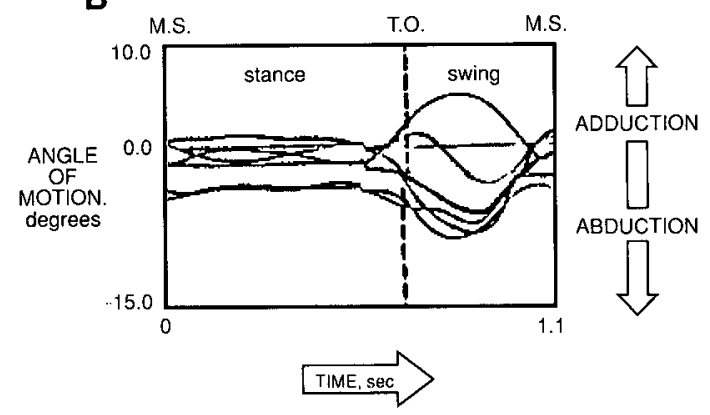

C

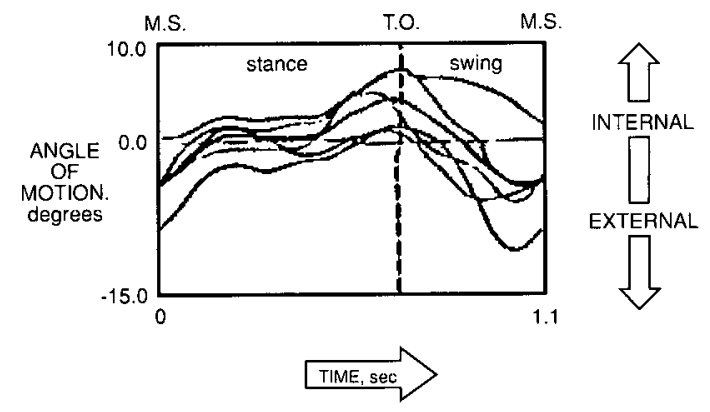

\section{TROCLEARTROSIS}

Su modelo mecánico se corresponde con el de una articulación en BISAGRA, con movimiento sobre un único eje. Anatómicamente está constituida por una superficie en forma de tróclea encajada en una superficie cóncova. Un ejemplo de esta unión articular en bisagra lo constituyen: el codo, la rodilla y el tobillo.

Aunque por su estructura anatómica estas articulaciones tendrían un sólo grado de libertad, en realidad no cumplen la premisa de moverse exclusivamente sobre un eje.

En un estudio tridimensional realizado «in vivo» sobre la cinemática de la rodilla, Lafortune y cols. ${ }^{3}$, demuestran cómo esta articulación realiza movimientos en los tres planos del espacio (Fig. 3).

Por otra parte, autores como $\mathrm{Chao}^{4}$, consideran la articulación del codo como una diartrosis con tres grados de libertad: flexión-extensión, abducción-adducción (marcada por la dirección de la polea humeral) y un tercer movimiento originado por la prono-supinación del antebrazo (Fig. 4).

En relación al tobillo, Murphy y cols. ${ }^{5}$, observan, en un estudio realizado sobre la dinámica de la articulación tibio-peroneo-astragalina en cadáveres, la existencia de un movimiento de rotación asociado a la flexión dorsoplantra que daba lugar a un segundo grado de libertad a la articulación del tobillo. Este concepto de movilidad biaxial del tobillo ha sido apoyado por los estudios dinámicos «in vivo» realizados por Scott y Winter6.

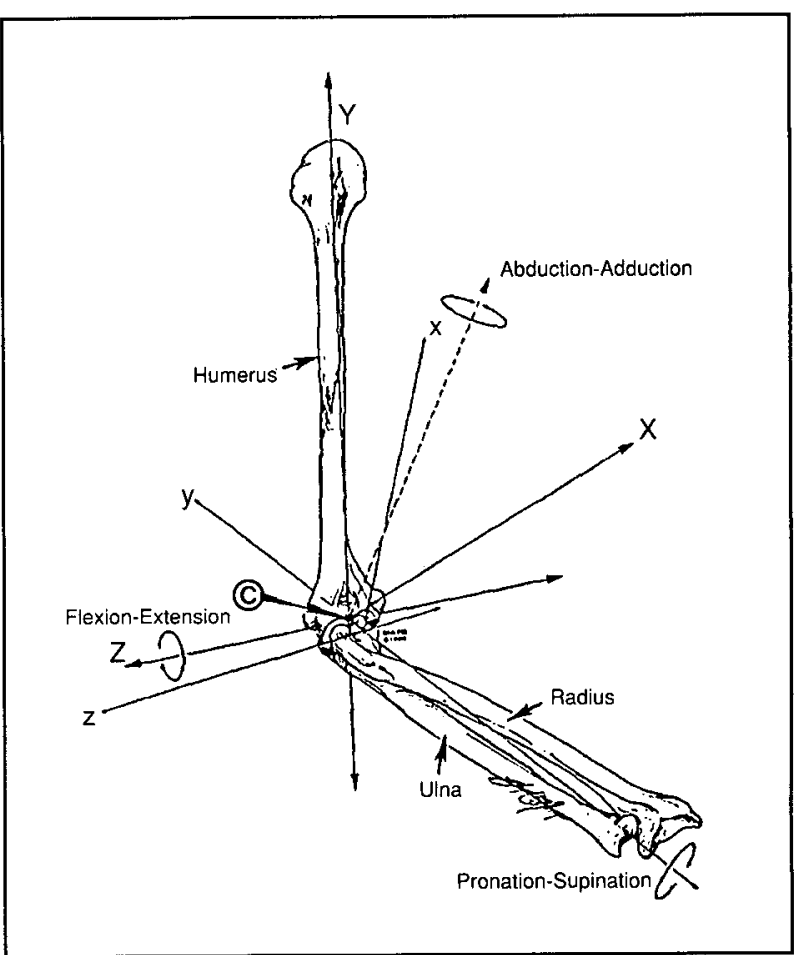

Fig. 4. Dibujo en el que se representan los movimientos realizados por la articulación del codo, y sus ejes correspondientes (Chao E.Y. y cols. J. Biomech. Egineering. 1980; 102: 301-310). 


\section{TROCOIDES}

Articulación establecida entre una superficie en forma de ARCO que se relaciona con una superficie CILINDRICA o eje central. El movimiento que realiza es uniaxial, y un ejemplo clásico de este tipo de articulación lo constituyen la unión entre el atlas y la apófisis odontoides del axis y la unión radio-cubital.

\section{CONDILOARTROSIS}

Denominada mecánicamente articulación ELIPSOIDAL, está constituida por la unión de una superficie condílea (ovoidea) que se sitúa o encaja en una cavidad elipsoidal. Posee movimiento biaxial.

Un ejemplo de este tipo de articulación es la unión húmero-radial, su diseño en forma de cóndilo va a permitir una discreta abducción-adducción del codo durante la flexo-extensión del mismo, originando el llamado "Carrying Angle» o tercer grado de libertad de movimiento del codo para autores como E. Chao ${ }^{4}$.

\section{ENCAJE RECIPROCO}

Superficie articular en forma de ENSILLADURA, incluida en una cavidad cóncavo-convexa en dirección opuesta.

El movimiento originado en este tipo de articulaciones es biaxial, similar a las articulaciones condíleas. Como ejemplo de este modelo articular podemos citar la unión establecida entre el primer metacarpino y el trapecio y, la articulación calcaneo-cuboidea.

\section{ARTRODIA}

Constituida generalmente por la unión de superficies articulares PLANAS, el movimiento que originan es el de deslizamiento. Este movimiento transcurre, según la anatomía clásica, sin posser un eje determinado de referencia, pero desde el punto de vista mecánico, Hartenbergs y Denavit ${ }^{7}$, describen cómo este tipo de articulaciones realizan movimientos de translación sobre dos ejes y de rotación sobre un tercero, aunque la amplitud del movimiento producido no sea lo suficientemente grande para ser observada a simple vista.
Como ejemplo de este tipo de articulaciones nos encontramos con las uniones establecidas entre los huesos del carpo y tarso.

Después de observar cómo los conceptos de movimiento atribuidos a las distintas diartrosis han sido modificados, en alguno de sus aspectos, por la aparición de nuevas técnicas de estudio y análisis de la cinemática articular, no debems olvidar que aunque la forma anatómica de los extremos óseos que constituyen la articulación influyen en gran manera en el movimiento que se origina en ella, las diferentes fuerzas que actúan sobre las estructuras articulares van a provocar una solicitación mecánica que, en mayor o menor grado, puede remodelar su diseño -como lo demuestra el hecho de la progresiva corrección del ángulo de anteversión e inclinación femoral que va modificándose desde el nacimiento hasta alcanzar la bipedestación y la marcha, no ocurre en aquellos niños que por presentar afectación del sistema nervioso central son incapaces de lograr la posición bípeda- existiendo una importante interrelación Forma-Función en el diseño anatómico articular, establecido por una flecha de doble sentido.

\section{BIBLIOGRAFIA}

1. Huson, A. Joints and Movements of the Foot: Terminology and Concepts. Acta Morphol Neerl-Scand, 1987; 25: $117-130$.

2. Owen, R., Goodfellow, J., Bullough, P. Fundamentos Científicos de Ortopedia y Traumatología. Salvat, S.A. Barcelona, Madrid. 1984.

3. Lafortune, M.A., Cavanagh, P.R., Sommer, H.J., Kalenak, A. Three-Dimensional Kinematics of the Human Knee During Walking. J Biomechanics, 1992; 25(4): 347-357.

4. Chao, E.Y., An, K.N., Askew, L.J., Morrey, B.F. Electrogoniometrer in elbow Joint Measurement. J Biomech Engineering, 1980; 102: 301-310.

5. Murphy, N., Allard, P., Aissaoui, R. Helical Motion Analysis of the Ankle. The second North American Congress of Biomechanics. Chicago, August 24th-28th, 1992.

6. Scott, S.H., Winter, D.A. Talocrural and Talocalcaneal Joint Kinematics and Kinetics During the Stance Phase of Walking. J Biomechanics, 1991; 24(8): 743-752.

7. Hartenbergs, R.S., Denavit, J. Kinematic Synthesis of Linkages. McGraw Hill. New York. 1964. 